\title{
ON REAL FIXED POINTS OF ONE PARAMETER FAMILY OF FUNCTION $x /\left(b^{x}-1\right)$
}

\author{
MOHAMMAD SAJID
}

\begin{abstract}
In the present paper, the real fixed points of one parameter family $\mathscr{T}=\left\{f_{\lambda}(x)\right.$ $=\lambda \frac{x}{b^{x}-1}$ and $\left.f_{\lambda}(0)=\frac{\lambda}{\ln b}: \lambda>0, x \in \mathbb{R}, b>0, b \neq 1\right\}$ are investigated. Further, the nature of these fixed points of $f_{\lambda}(x)$ are shown for $b>0$ except $b=1$.
\end{abstract}

Mostly, the dynamical behavior of functions play interesting role near to fixed points. The dynamics or iteration of polynomial, rational function or transcendental function without discontinuity points are simpler than transcendental functions with discontinuity points. Using the dynamics of functions near to real fixed points, the dynamics of functions in complex plane are induced by the following researchers: The dynamics of families of entire functions $\lambda \frac{\sinh (z)}{z}$ and $\lambda \frac{e^{z}-1}{z}, \lambda>0$ with infinitely many bounded singular values are studied by Prasad [6], and Kapoor and Prasad [4] respectively. Prasad and Nayak [7] also found the dynamics of certain class of critically bounded entire transcendental functions. The dynamics of $\lambda \frac{\sinh ^{2}(z)}{z^{4}}$ with infinitely many bounded singular values is found in [8] and the dynamics of certain transcendental meromorpsic functions with unbounded singular values is discussed in [5]. The dynamics of $\lambda e^{z}$ is found in [2]. The theory of fixed points of functions and its applications can be seen in [1,3].

A point $x$ is said to be a fixed point of function $f(x)$ if $f(x)=x$. A fixed point $x_{0}$ is called an attracting, neutral (indifferent) or repelling if $\left|f^{\prime}\left(x_{0}\right)\right|<1,\left|f^{\prime}\left(x_{0}\right)\right|=1$ or $\left|f^{\prime}\left(x_{0}\right)\right|>1$ respectively.

The present work describes the real fixed points of the function $\frac{x}{b^{x}-1}$ by considering one parameter family

$$
\mathscr{T}=\left\{f_{\lambda}(x)=\lambda \frac{x}{b^{x}-1} \text { and } f_{\lambda}(0)=\frac{\lambda}{\ln b}: \lambda>0, x \in \mathbb{R}, b>0, b \neq 1\right\} .
$$

Received December 4, 2013, accepted June 6, 2014.

2010 Mathematics Subject Classification. 37C25.

Key words and phrases. Fixed points. 
The present paper is organized as follows: In Theorem 1, the real fixed point of $f_{\lambda} \in \mathscr{T}$ are determined. The nature of these fixed points of $f_{\lambda}(x)$ are shown in Theorem 2.

For sake of convenience, let $f_{\lambda}(x)=\lambda f(x)$, where $f(x)=\frac{x}{b^{x}-1}$. Further, consider the function $\psi(x)=x f^{\prime}(x)+f(x)$. It is easily seen that the function $\psi(x)$ is continuous at $x=0$ and $\psi(0)=\frac{1}{\ln b}$.

\section{Lemma 1.}

(a) $\operatorname{For} 0<b<1$,

$$
\psi(x)=\frac{x}{\left(b^{x}-1\right)^{2}}\left[(2-x \ln b) b^{x}-2\right]\left\{\begin{array}{l}
<0 \text { for } x_{1}^{*}<x<0 \\
=0 \text { for } x=x_{1}^{*} \\
>0 \text { for } x<x_{1}^{*}
\end{array}\right.
$$

where $x_{1}^{*}$ is the unique negative real root of the equation $(2-x \ln b) b^{x}-2=0$.

(b) For $b>1$

$$
\psi(x)=\frac{x}{\left(b^{x}-1\right)^{2}}\left[(2-x \ln b) b^{x}-2\right]\left\{\begin{array}{l}
>0 \text { for } 0<x<x_{2}^{*} \\
=0 \text { for } x=x_{2}^{*} \\
<0 \text { for } x>x_{2}^{*}
\end{array}\right.
$$

where $x_{2}^{*}$ is the unique positive real root of the equation $(2-x \ln b) b^{x}-2=0$.

Proof. The function

$$
\psi(x)=x \frac{\left(b^{x}-1-x b^{x} \ln b\right)}{\left(b^{x}-1\right)^{2}}+\frac{x}{b^{x}-1}=\frac{x}{\left(b^{x}-1\right)^{2}}\left[(2-x \ln b) b^{x}-2\right]
$$

Suppose the function $r(x)=(2-x \ln b) b^{x}-2$. Then,

$$
r^{\prime}(x)=(1-x \ln b) b^{x} \ln b
$$

and

$$
r^{\prime \prime}(x)=-x b^{x}(\ln b)^{3}
$$

(i) For $0<b<1$

It is seen that, $x \in \mathbb{R}^{+}$,

$$
r^{\prime \prime}(x)>0
$$

By Equation (1.2), the function $r^{\prime}(x)$ is increasing on $\mathbb{R}^{+}$. Since $r^{\prime}(0)=\ln b, r^{\prime}(x) \rightarrow \infty$ as $x \rightarrow-\infty$ and $r^{\prime}(x)$ is continuous on $\mathbb{R}$, it gives that there is a unique $\tilde{x}_{1}<0$ such that $r^{\prime}(x)>0$ for $x<\tilde{x}_{1}, r^{\prime}\left(\tilde{x}_{1}\right)=0$ and $r^{\prime}(x)<0$ for $\tilde{x}_{1}<x$. Thus, $r(x)$ increases in $\left(-\infty, \tilde{x}_{1}\right)$, decreases in $\left(\tilde{x}_{1}, \infty\right)$ and attains its maximum at $\tilde{x}_{1}$. Since $r(0)=0$ and $r(x) \rightarrow-\infty$ as $x \rightarrow-\infty$, it conclude that there is a unique negative $x_{1}^{*}<\tilde{x}_{1}$ such that $r(x)<0$ for $-\infty<x<x_{1}^{*}, r\left(x_{1}^{*}\right)=0$ and $r(x)>0$ for $x_{1}^{*}<x<0$. Since $\frac{x}{\left(b^{x}-1\right)^{2}}<0$ for all $x<0$. Now, we have

$$
\psi(x)=\frac{x}{\left(b^{x}-1\right)^{2}} r(x)\left\{\begin{array}{l}
<0 \text { for } x_{1}^{*}<x<0 \\
=0 \text { for } x=x_{1}^{*} \\
>0 \text { for } x<x_{1}^{*}
\end{array}\right.
$$


where $x_{1}^{*}$ is the unique negative real root of the equation $(2-x \ln b) b^{x}-2=0$.

(ii) For $b>1$

It is seen that, for $x \in \mathbb{R}^{+}$,

$$
r^{\prime \prime}(x)<0
$$

By Equation (1.3), the function $r^{\prime}(x)$ is decreasing on $\mathbb{R}^{+}$. Since $r^{\prime}(0)=\ln b, r^{\prime}(x) \rightarrow-\infty$ as $x \rightarrow+\infty$ and $r^{\prime}(x)$ is continuous on $\mathbb{R}^{+}$, it shows that there is a unique $\tilde{x}_{2}>0$ such that $r^{\prime}(x)>0$ for $0 \leq x<\tilde{x}_{2}, r^{\prime}\left(\tilde{x}_{2}\right)=0$ and $r^{\prime}(x)<0$ for $x>\tilde{x}_{2}$. Therefore, $r(x)$ increases in [0, $\left.\tilde{x}_{2}\right)$, decreases in $\left(\tilde{x}_{2},+\infty\right)$ and attains its maximum at $\tilde{x}_{2}$. Since $r(0)=0$ and $r(x) \rightarrow-\infty$ as $x \rightarrow+\infty$, it establish that that there is a unique positive $x_{2}^{*}>\tilde{x}_{2}$ such that $r(x)>0$ for $0<x<x_{2}^{*}, r\left(x_{2}^{*}\right)=0$ and $r(x)>0$ for $x>x_{2}^{*}$. Since $\frac{x}{\left(b^{x}-1\right)^{2}}>0$ for all $x>0$. Now, it follows that

$$
\psi(x)=\frac{x}{\left(b^{x}-1\right)^{2}} r(x)\left\{\begin{array}{l}
>0 \text { for } 0<x<x_{2}^{*} \\
=0 \text { for } x=x_{2}^{*} \\
<0 \text { for } x>x_{2}^{*}
\end{array}\right.
$$

where $x_{2}^{*}$ is the unique positive real root of the equation $(2-x \ln b) b^{x}-2=0$.

Let us define

$$
\lambda^{*}=\frac{x^{*}}{f\left(x^{*}\right)}=b^{x^{*}}-1
$$

where $x^{*}$ is the unique real root of the equation $(2-x \ln b) b^{x}-2=0$. The root $x^{*}$ is negative if $0<b<1$ and positive if $b>1$.

The following theorem shows that the function $f_{\lambda}(x)$ has a unique real fixed point:

Theorem 1. Let $f_{\lambda} \in \mathscr{T}$. Then, the function $f_{\lambda}(x)$ has a unique real fixed point $x_{\lambda}$. The fixed point $x_{\lambda}$ is negative if $0<b<1$ and positive if $b>1$.

Proof. Since $f_{\lambda}(x)<0$ if $0<b<1$ and $f_{\lambda}(x)>0$ if $b>1$ for all $x \in \mathbb{R}$, each real fixed point of $f_{\lambda}(x)$ is negative for $0<b<1$ and positive for $b>1$. For $x \in \mathbb{R}$ and $b>0$ except $b=1$, $f_{\lambda}^{\prime}(x)=\lambda \frac{(1-x \ln b) b^{x}-1}{\left(b^{x}-1\right)^{2}}<0$.

Let $h_{\lambda}(x)=f_{\lambda}(x)-x$ for $x \in \mathbb{R}$. Since the function $f_{\lambda}^{\prime}(x)$ is continuous at $x=0, f_{\lambda}^{\prime}(0)=-\frac{\lambda}{2}$ and $f_{\lambda}^{\prime}(x)<0$ for $x \in \mathbb{R}, b>0$ except $b=1$, then $h_{\lambda}^{\prime}(x)=f_{\lambda}^{\prime}(x)-1<0$. Hence, the function $h_{\lambda}(x)$ is decreasing. Moreover, (i) for $0<b<1, h_{\lambda}(0)=\frac{\lambda}{\ln b}<0, h_{\lambda}(x) \rightarrow+\infty$ as $x \rightarrow-\infty$ and $h_{\lambda}(x)$ is continuous on $\mathbb{R}^{-}$, (ii) $h_{\lambda}(0)=\frac{\lambda}{\ln b}>0, h_{\lambda}(x) \rightarrow-\infty$ as $x \rightarrow+\infty$ and $h_{\lambda}(x)$ is continuous on $\mathbb{R}^{+}$, by the intermediate value theorem, there exists a unique $x_{\lambda}$ such that $h_{\lambda}\left(x_{\lambda}\right)=0$. Thus, it follows that $f_{\lambda}(x)$ has a unique real fixed point $x_{\lambda}$.

Remark 1. For $\lambda>\lambda^{*}$, there may exist periodic points of period greater than or equal to 2 . This case is not considered here. It is left for forthcoming work. 
In the following theorem, the nature of fixed points of $f_{\lambda}(x)$ are determined:

Theorem 2. Let $f_{\lambda} \in \mathscr{T}$. Then, the fixed point $x_{\lambda}$ of the function $f_{\lambda}(x)$ is (i) an attracting for $0<\lambda<\lambda^{*}$ (ii) rationally indifferent for $\lambda=\lambda^{*}$ (iii) repelling for $\lambda>\lambda^{*}$.

Proof. Since the derivative of $\frac{x}{f(x)}$ is negative for $x \in \mathbb{R}^{-}, 0<b<1$ and is positive for $x \in \mathbb{R}^{+}$, $b>1$, the function $\frac{x}{f(x)}$ is decreasing on $\mathbb{R}^{-}$for $0<b<1$ and is increasing on $\mathbb{R}^{+}$for $b>1$. We prove the following cases for $0<b<1$ :

(i) For $0<\lambda<\lambda^{*}$, since the function $\frac{x}{f(x)}$ is decreasing on $\mathbb{R}^{-}$and $\lambda=\frac{x_{\lambda}}{f\left(x_{\lambda}\right)}$, we have $\frac{x_{\lambda}}{f\left(x_{\lambda}\right)}<$ $\frac{x^{*}}{f\left(x^{*}\right)}$. It means that $b^{x_{\lambda}}<b^{x^{*}}$. Hence, $x_{\lambda}>x^{*}$. By Lemma $1(\mathrm{a}), \psi\left(x_{\lambda}\right)<0$. Since $f_{\lambda}^{\prime}\left(x_{\lambda}\right)=$ $\frac{\psi\left(x_{\lambda}\right)}{f\left(x_{\lambda}\right)}-1$, it follows that $f_{\lambda}^{\prime}\left(x_{\lambda}\right)+1=\frac{\psi\left(x_{\lambda}\right)}{f\left(x_{\lambda}\right)}>0$. Since $f_{\lambda}^{\prime}(x)$ is negative on $\mathbb{R}^{-}$, it shows that $-1<f_{\lambda}^{\prime}\left(x_{\lambda}\right)<0$ and consequently, the fixed point $x_{\lambda}$ of $f_{\lambda}(x)$ is an attracting for $0<\lambda<\lambda^{*}$.

(ii) For $\lambda=\lambda^{*}$, it is easy to prove $x_{\lambda}=x^{*}$. Now, by Lemma 1(a), it follows that $f_{\lambda}^{\prime}\left(x_{\lambda}\right)+1=$ $\frac{\psi\left(x_{\lambda}\right)}{f\left(x_{\lambda}\right)}=0$ which implying $f_{\lambda^{*}}^{\prime}\left(x_{\lambda}\right)=-1$. Therefore, the fixed point $x^{*}$ of $f_{\lambda}(x)$ is rationally indifferent for $\lambda=\lambda^{*}$.

(iii) For $\lambda>\lambda^{*}$, by similar arguments used in (i), it follows that $x_{\lambda}<x^{*}$. Again, by Lemma 1(a), we have $\psi\left(x_{\lambda}\right)>0$. It gives that $f_{\lambda}^{\prime}\left(x_{\lambda}\right)+1=\frac{\psi\left(x_{\lambda}\right)}{f\left(x_{\lambda}\right)}<0$ and hence $f_{\lambda}^{\prime}\left(x_{\lambda}\right)<-1$. Therefore, $x_{\lambda}$ is repelling fixed point of $f\left(x_{\lambda}\right)$ for $\lambda>\lambda^{*}$.

For $b>1$, by using Lemma 1(b), the proof of all cases are similar and easily derived as above.

It is observed that, from Theorem 2, the nature of the fixed point changes when parameter $\lambda$ crosses parameter value $\lambda^{*}$.

\section{Acknowledgement}

The author is thankful to referees for careful reviewing and providing very valuable suggestions to improve this paper.

\section{References}

[1] Alan F. Beardon, Iteration of Rational Functions, Springer, New York, 2000.

[2] R. L. Devaney, $e^{z}$ - Dynamics and Bifurcation, International Journal of Bifurcations and Chaos, 1 (1991), 287-308.

[3] X. H. Hua and C. C. Yang, Dynamics of Transcendental Functions, Gordan and Breach Sci. Pub., Amsterdam, 1998.

[4] G. P. Kapoor and M. Guru Prem Prasad, Dynamics of $\left(e^{z}-1\right) / z$ : the Julia set and Bifurcation, Ergodic Theory and Dynamical Systems, 18(6) (1998), 1363-1383.

[5] Tarakanta Nayak and M. Guru Prem Prasad, Iteration of certain meromorphic functions with unbounded singular values, Ergodic Theory and Dynamical Systems, 30(3) (Jun 2010), 877-891. 
[6] M. Guru Prem Prasad, Chaotic burst in the dynamics of $f_{\lambda}(z)=\lambda \frac{\sinh (z)}{z}$, Regular and Chaotic Dynamics, 10(1) (2005), 71-80.

[7] M. Guru Prem Prasad and Tarakanta Nayak, Dynamics of certain class of critically bounded entire transcendental functions, J. Math. Anal. Appl., 329(2) (2007), 1446-1459.

[8] M. Sajid and G. P. Kapoor, Dynamics of a family of non-critically finite even transcendental meromorphic functions, Regular and Chaotic Dynamics, 9(2) (2004), 143-162.

College of Engineering, Qassim University, Buraidah, Al-Qassim, Saudi Arabia.

E-mail: msajid@qec.edu.sa

E-mail: msajid.in@gmail.com 